kinds of homomorphisms (join, meet, lattice, order) is remarkable for its completeness and clarity. There are a few exercises, but they are not very numerous or exciting. The book is divided into five chapters, as follows: (I) Foundations; (II) The simplest classes of lattices; (III) Modular lattices; (IV) Distributive and Boolean lattices; (V) Miscellany. The fifth chapter discusses Zorn's lemma, congruence relations, and some connections between Boolean algebra and logic. There is an appendix that treats in great generality the universal algebraic concepts (e.g., homomorphism) of which special cases are treated in the body of the book. There is nothing new here for the expert, but a beginning student might find the treatment a pleasant illustration of some of the easier techniques of algebra.

\title{
Paul R. Halmos
}

\section{RESEARCH PROBLEMS}

7. H. E. Salzer: Numerical analysis.

It is known that $\mathrm{I}$ : an $n$th degree polynomial is determined by $(n+1)$ values, considering its value and the first consecutive $r$ derivatives at a point as $(r+1)$ values. The writer found for $(2 n-1)$ th degree formulas of the type (1) $f_{[n / 2]+1}=\sum_{i=-[(n-1) / 2]}^{[n / 2]}$ - $\left(A_{i} f_{i}+h^{2} B_{i} f_{i}^{\prime \prime}\right)$ the existence of solutions $A_{i}, B_{i}$ for $n=2$ and 4 , but not for $n=1$ (trivial), 3 , or 5 , suggesting the following:

(a) In general is a polynomial of $(2 n-1)$ th degree determined by its value and its second derivative at $n$ equally-spaced points if and only if $n$ is even?

(b) What generalizations of I are there, involving $(r+1)$ non-consecutive derivatives (value considered as 0 th derivative)? Thus the writer found $(2 n)$ th degree formulas of the type (2) $f_{[n / 2]+1}=\sum_{i=-[(n-1) / 2]}^{[n / 2]} A_{i} f_{i}+h \sum_{i=-[n-1) / 2]}^{[n / 2]+1} B_{i} f_{i}^{\prime}$ for $n=1(1) 5$, indicating that $f$ can be determined from $n$ values of $f$ and $f^{\prime}$ at $n$ points and $f^{\prime}$ at an $(n+1)$ th point. (Received December 5, 1955.)

\section{H. E. Salzer: Numerical analysis.}

Theintegrationformula $(1) \int^{1}-1\left(f(x) /\left(1-x^{2}\right)^{1 / 2}\right) d x=(\pi / n)[f(\cos \pi / 2 n)+f(\cos 3 \pi / 2 n)$ $+\cdots+f(\cos (2 n-1) \pi / 2 n)]$ is exact for $f(x)$ any $(2 n-1)$ th degree polynomial, so that the weight function $1 /\left(1-x^{2}\right)^{1 / 2}$ gives a Gaussian-type quadrature formula for a finite interval, which happens to be equally-weighted. Can one find weight functions $w(x)$ and $W(x)$ yielding Gaussian-type quadrature formulas of the form (2) $\int_{0}^{\infty} w(x) f(x) d x=(a / n) \sum_{i=1}^{n} f\left(x_{i}\right)$ and $(3) \int_{-\infty}^{\infty} W(x) f(x) d x=(b / n) \sum_{i=1}^{n} f\left(x_{i}\right)$, combining the advantage of orthogonality or algebraic degree of precision equal to $2 n-1$ with equal weights? Unlike (1), do solutions to (2) and (3) exist only when $w(x)$ and $W(x)$ depend upon $n$ ? (Received December 5, 1955.) 